\title{
LA ESTABILIDAD DE LA FUNCIONAL CUADRÁTICA-ADITIVA EN UN ESPACIO DE BANACH NO-ARQUIMEDIANO
}

\author{
Alex Armando Cruz Huallpara ${ }^{1}$, Josué Alonso Aguirre Enciso ${ }^{2}$, \\ Luis Miguel Nuñez Ramirez ${ }^{3}$, Rodolfo Jose Galvez Perez ${ }^{4}$, \\ Humberto Emiliano Galvez Perez,
}

Resumen: En este trabajo presentamos el estudio de la estabilidad generalizada de Hyers-Ulam, de la ecuación funcional cuadrática-aditiva en un espacio de Banach No-Arquimediano, utilizando el método directo y del punto fijo.

Palabras clave: Espacio de Banach No-Arquimediano , aplicación aditiva, aplicación cuadrática, método del punto fijo.

\section{STABILITY OF ADDITIVE - QUADRATIC MAPPINGS IN BANACH SPACE NON-ARCHIMEDEAN}

\begin{abstract}
In this paper we study the generalized Hyers-Ulam stability of the quadratic-additive functional equation in a Non-Archimedean Banach space, using the direct method and the fixed point.
\end{abstract}

Keywords: Non-Archimedean Banach space, additive application, quadratic application, fixed point method.

\section{Introducción}

En el estudio de la teoría de ecuaciones funcionales se plantea la siguiente interrogante: ¿Qué condiciones debe de cumplir una función que satisface (aproximadamente) una ecuación funcional, para que sea una solución exacta de dicha ecuación funcional?, el primer estudio que se realizo para dar respuesta a esta pregunta lo hizo Ulam con el problema de la estabilidad relativa de grupo de homomorfismos, en el año 1940; luego en el siguiente año, Hyers generalizo el estudio para aplicaciones aditivas. Este nuevo concepto se conoce como la estabilidad generalizada de Hyers-Ulam; en 1983 Skof demostro el problema de la estabilidad generalizada de Hyers-Ulam para las ecuaciones funcionales cuadráticas de las aplicaciones $f: X \longrightarrow Y$, donde $X$ es un espacio normado e $Y$ es un espacio de Banach, luego en 2002 Czerwik demuestra la estabilidad generalizada Hyers-Ulam de ecuaciones funcionales cuadráticas, en este articulo se realiza una revisión de los temas más importantes de esta teoría.

\footnotetext{
${ }^{1}$ UNMSM, Facultad de Ciencias Matemáticas, e-mail: acruzh@unmsm.edu.pe

${ }^{2}$ UNMSM, Facultad de Ciencias Matemáticas, e-mail:josuea.aguirre@gmail.com

${ }^{3}$ UNMSM, Facultad de Ciencias Matemáticas, e-mail:lnuñezr@unmsm.edu.pe

${ }^{4}$ UNMSM, Facultad de Ciencias Matemáticas, e-mail: rgalvezp@unmsm.edu.pe

${ }^{5}$ UNMSM, Facultad de Ciencias Matemáticas, e-mail:hgalvezp@unmsm.edu.pe
} 


\section{Definiciones y Resultados Preliminares}

Definición 2.1 Sea $\mathbb{K}$ un cuerpo, una valuación en un cuerpo $\mathbb{K}$ es una aplicación:

$$
|.|: \mathbb{K} \longrightarrow \mathbb{R}
$$

tal que para cada $x, y \in \mathbb{K}$ satisface las siguientes condiciones :

i) $|x| \geq 0$

ii) $|x|=0$ si, $y$ sólo si $x=0$

iii) $|x+y| \leq|x|+|y|$ (Desigualdad Triangular)

el par $(\mathbb{K},||$.$) es llamado cuerpo valuado.$

Definición 2.2 Sea $(\mathbb{K},||$.$) un cuerpo valuado.$

i) Se llama valuación Arquimediana, si $\left|n \cdot 1_{\mathbb{K}}\right|>1$ para algún $n \in \mathbb{N}$

ii) Se llama valuación No-Arquimediana, si $\left|n \cdot 1_{\mathbb{K}}\right| \leq 1$ para todo $n \in \mathbb{N}$

donde $1_{\mathbb{K}}$ es el elemento identidad del cuerpo $\mathbb{K}$.

Definición 2.3 Un cuerpo es llamado No Arquimediano, si la valuación del cuerpo es NoAquimediana.

Proposición 2.4 Sea $\mathbb{K}$ un cuerpo valuado, las siguientes afirmaciones son equivalentes

i) $\mathbb{K}$ es un cuerpo No Arquimediano

ii) Para todo $a, b \in \mathbb{K}$ se tiene que $|a+b| \leq \max \{|a|,|b|\}$ (Desigualdad Triangular Fuerte)

Prueba. (Ver [2]).

Proposición 2.5 Sea $\mathbb{K}$ un cuerpo No Arqimediano. Si $a, b \in \mathbb{K} y|a|<|b|$, entonces $|b-a|=|b|$

Prueba. (Ver [9]).

Definición 2.6 Sea $X$ un espacio vectorial sobre $(\mathbb{K},||$.$) un cuerpo No Arquimediano. Una$ norma No-arquimediana en $X$ es una aplicación

$$
\|\cdot\|: X \longrightarrow \mathbb{R}
$$

tal que para cada $x, y \in X$ y dado, $\lambda \in \mathbb{K}$ satisfacen las siguientes condiciones:

i) $\|x\| \geq 0$

ii) $\|x\|=0$ si, $y$ sólo si $x=0$

iii) $\|\lambda \cdot x\|=|\lambda| \cdot|| x||$

iv) $\|x+y\| \leq \max \{\|x\|,\|y\|\}$ (Desigualdad Triangular Fuerte)

Definición 2.7 Sea $(X,\|\|$.$) un espacio No-Arquimediano. Sea r \in \mathbb{K}, a \in X$ definimos los siguientes conjuntos:

i) $B_{r}(a)=\{x \in X ;\|x-a\|<a\}$ es la bola abierta de centro a y radio $r$

i) $B_{r}[a]=\{x \in X ;\|x-a\| \leq a\}$ es la bola cerrada de centro a y radio $r$ 
Proposición 2.8 Sea $(X,\|\|$.$) un espacio No-Arquimediano$

i) $B_{r}[a]$ es un conjunto abierto y cerrado

ii) $B_{\epsilon}[a] \cap B_{\epsilon}\left[a^{\prime}\right] \neq \phi$ entonces $B_{\epsilon}[a]=B_{\epsilon}\left[a^{\prime}\right]$

iii) $B, B^{\prime}$ bolas cerradas, con $B \cap B^{\prime}=\phi$ entonces $B \subset B^{\prime}$ o $B^{\prime} \subset B$

Prueba. (Ver [2]).

\section{Observación 2.9}

i) La Proposición 2.8. también es válida para bolas abiertas. Por lo cual es un espacio totalmente disconexo.

ii) De la Proposición 2.8.(ii) se tiene que las bolas no dependen del centro.

iii) De la Proposición 2.8.(iii) si dos bolas tienen al menos un punto en común entonces una bola está incluida en la otra.

iv) Sea $X$ un espacio No-Arquimediano, $\left\{x_{n}\right\}$ una sucesión en $X$ entonces $\left\|x_{n}-x_{m}\right\| \leq \max \left\{\left\|x_{j+1}-x_{j}\right\| ; m \leq j \leq n-1\right\}$ donde $(n>m)$

Definición 2.10 Sea $\left\{x_{n}\right\}$ una sucesión en un espacio No.Arquimediano:

i) Una sucesión $\left\{x_{n}\right\}$ es de Cauchy si, y sólo si $\left\{x_{n+1}-x_{n}\right\}$ converge a cero en un espacio No-Arquimediano

ii) Un espacio No-Arquimediano es completo si toda sucesión de Cauchy es convergente.

Teorema 2.11 Sea $(X, d)$ un espacio métrico completo y sea $J: X \longrightarrow X$ una aplicación estrictamente contractil con constante de Lipschitz $\alpha<1$. Entonces para cada $x \in X$

$$
d\left(J^{n} x, J^{n+1} x\right)=\infty
$$

para todos los enteros $n$ no negativos, existe un entero positivo $n_{0}$ tal que

1) $d\left(J^{n} x, J^{n+1} x\right)<\infty, \forall n \geq n_{0}$

2) la sucesión $J^{n} x$ converge a un punto fijo $y^{*}$ de $J$

3) $y^{*}$ es el único punto fijo de $J$ en el conjunto $Y=\left\{y \in X ; d\left(J^{n_{0}} x, y\right)<\infty\right\}$

4) $d\left(y, y^{*}\right) \leq \frac{1}{1-\alpha} d(y, J y)$ para todo $y \in Y$

Prueba. (Ver [6]).

En 1996 , G. Isac y M. Rassias fueron los primeros en ofrecer aplicaciones de la teoría de la estabilidad de las ecuaciones funcionales para la prueba de nuevos teoremas de punto fijo con aplicaciones. Mediante el uso del método del punto fijo, los problemas de estabilidad de varias ecuaciones funcionales se han investigado extensamente por varios autores. En este trabajo se utiliza el método del punto fijo y método directo, se demuestra la estabilidad Hyers-Ulam de la siguiente ecuación funcional cuadrática -aditiva

$$
\begin{gathered}
r f\left(\frac{x+y+z}{s}\right)+r f\left(\frac{x-y+z}{s}\right)+r f\left(\frac{x+y-z}{s}\right)+r f\left(\frac{-x+y+z}{s}\right) \\
=\gamma f(x)+\gamma f(y)+\gamma f(z)
\end{gathered}
$$

donde $x, y, z \in X$, siendo $X$ un espacio normado No-Arquimediano. Es fácil ver que una aplicación $f$ con $f(0)=0$ es una solución de la ecuación (1) si y sólo si $f$ es de la forma $f(x)=A(x)+Q(x)$ para todo $x \in X$. 


\section{Estabilidad del Funcional de la Ecuación (1): Método del Punto Fijo}

Teorema 3.1 Sea $X$ un espacio normado No-Arquimediano e $Y$ un espacio completo NoArquimediano. Sea $\varphi: X^{3} \longrightarrow[0, \infty)$ una función tal que existe un $\alpha<1$ con

$$
\varphi(2 x, 2 y, 2 z) \leq|2| \alpha \varphi(x, y, z)
$$

para todo $x, y, z \in X$. Sea $f: X \longrightarrow Y$ una aplicación que satisface

$$
\begin{aligned}
\| r^{2} f\left(\frac{x+y+z}{s}\right)+ & r^{2} f\left(\frac{x-y+z}{s}\right)+r 2 f\left(\frac{x+y-z}{s}\right)+ \\
& r^{2} f\left(\frac{-x+y+z}{s}\right)-4 f(x)-4 f(y)-4 f(z) \|_{Y} \leq \varphi(x, y, z)
\end{aligned}
$$

para todo $x, y, z \in X$. Entonces existe un única aplicación aditiva $A: X \longrightarrow Y$ tal que

$$
\|f(x)-A(x)\|_{Y} \leq \frac{\max \{\varphi(2 x, 0,0), \varphi(x, x, 0)\}}{|2 \gamma|(1-\alpha)} \text { para todo } x \in X
$$

Demostración. Para $y=z=0$ en (3) y reemplazar $x$ por $2 x$, obtenemos

$$
\left\|r f\left(\frac{2 x}{s}\right)-\frac{\gamma}{2} f(x)\right\|_{Y} \leq \frac{1}{|2|} \varphi(2 x, 0,0)
$$

para todo $x \in X$. Para $y=x$ y en (3), tenemos

$$
\left\|r f\left(\frac{2 x}{s}\right)-\gamma f(x)\right\|_{Y} \leq \frac{1}{|2|} \varphi(x, x, 0)
$$

para todo $x \in X$. Por (5), obtenemos

$$
\begin{aligned}
&\left\|\frac{f(2 x)}{2}-f(x)\right\|_{Y}=\frac{1}{|\gamma|}\left\|\frac{\gamma}{2} f(2 x) \pm r f\left(\frac{2 x}{s}\right)-\gamma f(x)\right\|_{Y} \\
& \leq \frac{1}{|\gamma|} \max \left\{\left\|r f\left(\frac{2 x}{s}\right)-\frac{\gamma}{2} f(2 x)\right\|\left\|_{Y},\right\| r f\left(\frac{2 x}{s}\right)-\gamma f(x) \|_{Y}\right\} \\
& \leq \frac{1}{|2 \gamma|}\{\varphi(2 x, 0,0), \varphi(x, x, 0)\}
\end{aligned}
$$

Definimos el conjunto $S=\{h: X \longrightarrow Y\}$ donde $X$ un espacio normado No-Arquimediano e $Y$ es un espacio completo No-Arquimediano. Introducimos una métrica en $S$ de la forma :

$$
d(g, h)=\inf \left\{\mu \in(0,+\infty) ;\|g(x)-h(x)\|_{Y} \leq \mu \cdot \max \{\varphi(2 x, 0,0), \varphi(x, x, 0)\}, \forall x \in X\right\}
$$

donde, inf $\phi=+\infty$. Es evidente que $(S, d)$ es completo. Ahora definimos la aplicación lineal $J: S \longrightarrow S$ tal que $J g(x)=\frac{1}{2} g(2 x)$ para todo $x \in X$. Sea $g, h \in S$ tales que $d(g, h)=\varepsilon$. Entonces

$$
\|g(x)-h(x)\|_{Y} \leq \epsilon \cdot \max \{\varphi(2 x, 0,0), \varphi(x, x, 0)\} \text { para todo } x \in X .
$$

Por lo tanto,

$$
\begin{aligned}
\|J g(x)-J h(x)\|_{Y} & =\left\|\frac{1}{2} g(2 x)-\frac{1}{2} h(2 x)\right\| \|_{Y}=\frac{\|g(2 x)-h(2 x)\|_{Y}}{|2|} \\
& \leq \frac{\epsilon}{|2|} \max \{\varphi(4 x, 0,0), \varphi(2 x, 2 x, 0)\} \leq \alpha \cdot \epsilon \cdot \max \{\varphi(2 x, 0,0), \varphi(x, x, 0)\}
\end{aligned}
$$


Para todo $x \in X$. De este modo, si $d(g, h)=\varepsilon$ entonces $d(J g, J h) \leq \alpha \varepsilon$. Esto es, $d(J g, J h) \leq$ $\alpha d(g, h)$ para todo $g, h \in S$.

Además por $(6)$ se deduce que $d(J g, J h) \leq \frac{1}{|2 y|}$. Luego por el Teorema 2.11. existe una aplicación $A: X \longrightarrow Y$ que satisface lo siguiente:

(i) $A$ es un punto fijo de $J$, es decir,

$$
2 A(x)=A(2 x) \text { para todo } x \in X .
$$

$A$ es un único punto fijo de $J$ en el conjunto $M=\{g \in S ; d(h, g)<\infty\}$, esto implica que $A$ es un única aplicación que satisface (7) tal que para algún $\mu \in(0,+\infty)$ satisface $\|f(x)-A(x)\|_{Y} \leq \mu \cdot \max \{\varphi(2 x, 0,0), \varphi(x, x, 0)\}$ para todo $x \in X$.

(ii) $d\left(J^{n} f, A\right) \longrightarrow 0$ cuando $n \longrightarrow \infty$. Entonces

$$
\lim _{n \rightarrow \infty} \frac{f\left(2^{n} x\right)}{2^{n}}=A(x) \text { para todo } x \in X
$$

(iii) $d(f, A) \leq \frac{1}{1-\alpha} d(f, J f)$, esto es $d(f, A) \leq \frac{1}{|2 \gamma|(1-\alpha)}$, lo cual nos dice que la ecuación (3) es invariante.

Luego se deduce de (1) y (2) que

$$
\begin{aligned}
& \| r A\left(\frac{x+y+z}{s}\right)+r A\left(\frac{x-y+z}{s}\right)+r A\left(\frac{x+y-z}{s}\right)+r A\left(\frac{-x+y+z}{s}\right)- \\
& \gamma A(x)-\gamma A(y)-\gamma A(z) \|_{Y} \\
& =\lim _{n \rightarrow \infty} \frac{1}{|2|^{n}} \| r f\left(\frac{2^{n}(x+y+z)}{s}\right)+r f\left(\frac{2^{n}(x-y+z)}{s}\right)+r f\left(\frac{2^{n}(x+y-z)}{s}\right)+ \\
& r f\left(\frac{2^{n}(-x+y+z)}{s}\right)-\gamma f\left(2^{n} x\right)-\gamma f\left(2^{n} y\right)-\gamma f\left(2^{n} z\right) \|_{Y} \\
& \leq \lim _{n \longrightarrow \infty} \frac{1}{|2|^{n}} \varphi\left(2^{n} x, 2^{n} y, 2^{n} z\right) \leq \lim _{n \longrightarrow \infty} \frac{1}{|2|^{n}}|2|^{n} \alpha^{n} \varphi(x, y, z) \text { para todo } x, y, z \in X
\end{aligned}
$$

Así

$r A\left(\frac{x+y+z}{s}\right)+r A\left(\frac{x-y+z}{s}\right)+r A\left(\frac{x+y-z}{s}\right)+r A\left(\frac{-x+y+z}{s}\right)-\gamma A(x)-\gamma A(y)-\gamma A(z)=0$

para todo $x, y, z \in X$. Por tanto, $A: X \longrightarrow Y$ satisface (1) y esto completa la prueba.

Corolario 3.2 Sea $\theta$ un número real positivo y $q$ un número real con $q>1$. Sea $f: X \longrightarrow Y$ una aplicación que satisface

$$
\begin{aligned}
& \| r f\left(\frac{x+y+z}{s}\right)+r f\left(\frac{x-y+z}{s}\right)+r f\left(\frac{x+y-z}{s}\right)+ \\
& \quad r f\left(\frac{-x+y+z}{s}\right)-\gamma f(x)-\gamma f(y)-\gamma f(z) \|_{Y} \leq \theta\left(\|x\|^{q}+\|y\|^{q}+\|z\|^{q}\right)
\end{aligned}
$$

para todo $x, y, z \in X$. Entonces existe una única aplicación aditiva $A: X \longrightarrow Y$ tal que

$$
\|f(x)-A(x)\|_{Y} \leq \frac{\left.2|2| \theta|| x\right|^{q}}{|2 \gamma|\left(|2|-|2|^{q}\right)} \text { para todo } x \in X
$$


Prueba. La prueba se sigue del Teorema 3.1, tomando $\varphi(x, y, z)=\theta\left(\|x\|^{q}+\|y\|^{q}+\|z\|^{q}\right)$ para $x, y, z \in X$; entonces podemos elegir $\alpha=|2|^{q-1}$ y obtenemos el resultado.

Teorema 3.3 Sea $X$ un espacio normado No-Arquimediano e $Y$ un espacio completo NoArquimediano. Sea $\varphi: X^{3} \longrightarrow[0,+\infty)$ un funcional tal que existe $\alpha<1$ con

$$
\varphi\left(\frac{x}{2}, \frac{y}{2}, \frac{z}{2}\right) \leq \frac{\alpha}{|2|} \varphi(x, y, z) \text { para todo } x, y, z \in X .
$$

Sea $f: X \longrightarrow Y$ una aplicación que satisface (2). Entonces existe una única aplicación aditiva $A: X \longrightarrow Y$ tal que

$$
\|f(x)-A(x)\|_{Y} \leq \frac{\alpha \cdot \max \{\varphi(2 x, 0,0), \varphi(x, x, 0)\}}{|2 \gamma|(1-\alpha)} \text { para todo } x \in X
$$

Demostración. Sea $(S, d)$ un espacio métrico definido en la prueba del Teorema 3.1. Consideremos la aplicación $J: S \longrightarrow S$ tal que

$$
J g(x):=2 g\left(\frac{x}{2}\right) \text { para todo } x \in X .
$$

Reemplazando $x$ por $\frac{x}{2}$ en (6) y utilizar (9) tenemos que

$$
\left\|f(x)-2 f\left(\frac{x}{2}\right)\right\|_{Y} \leq \frac{1}{|\gamma|} \max \left\{\varphi(x, 0,0), \varphi\left(\frac{x}{2}, \frac{x}{2}, 0\right) \leq \frac{\alpha}{|2 \gamma|} \max \{\varphi(2 x, 0,0), \varphi(x, x, 0)\}\right.
$$

Asi $d(f, J f) \leq \frac{\alpha}{|2 y|}$ el resto de la prueba es similar al Teorema 3.1.

Corolario 3.4 Sea $\theta$ un número real positivo y $q$ es un numero real con $0<q<1$. Sea $f: X \longrightarrow Y$ una aplicación que satisface (8). entonces existe una única aplicación aditiva $A: X \longrightarrow Y$ tal que

$$
\|f(x)-A(x)\|_{Y} \leq \frac{\left.2|2| \theta|| x\right|^{q}}{|2 \gamma|\left(|2|^{q}-|2|\right)} \text { para todo } x \in X
$$

Prueba. La prueba se sigue del Teorema 3.3. tomando $\varphi(x, y, z)=\theta\left(\|x\|^{q}+\|y\|^{q}+\|z\|^{q}\right)$ para todo $x, y, z \in X$. Entonces podemos elegir $\alpha=|2|^{1-q}$ y obtenemos el resultado deseado.

Teorema 3.5 Sea $X$ un espacio normado No-Arquimediano e $Y$ un espacio completo NoArquimediano. Sea $\varphi: X^{3} \longrightarrow[0,+\infty)$ un funcional tal que existe un $\alpha<1$ con

$$
\varphi(2 x, 2 y, 2 z) \leq|4| \alpha \varphi(x, y, z) \text { para todo } x, y, z \in X .
$$

Sea $f: X \longrightarrow Y$ una aplicación con $f(0)=0$ y satisface (2). Entonces existe una única aplicación cuadrática $Q: X \longrightarrow Y$ tal que

$$
\|f(x)-Q(x)\|_{Y} \leq \frac{\max \{\varphi(2 x, 0,0),|2| \varphi(x, x, 0)\}}{|4 \gamma|(1-\alpha)} \text { para todo } x \in X .
$$

Demostración. Considremos el conjunto $S^{*}=\{g: X \longrightarrow Y ; g(0)=0\}$ y la métrica $d^{*}$ en $S^{*}$ definido por

$$
d^{*}(g, h)=\inf \left\{\mu \in(0,+\infty) ;\|g(x)-h(x)\|_{Y} \leq \mu \cdot \max \{\varphi(2 x, 0,0),|2| \varphi(x, x, 0)\}, \forall x \in X\right\}
$$

como $\inf \emptyset=+\infty$. Es fácil mostrar que $\left(S^{*}, d^{*}\right)$ es completo . Considerar la aplicación lineal $J:\left(S^{*}, d^{*}\right) \longrightarrow\left(S^{*}, d^{*}\right)$ tal que

$$
J g(x)=\frac{1}{4} g(2 x), \text { para todo } x \in X
$$


reemplazando $y=x$ y $z=0$ en $(2)$, tenemos que

$$
\left\|2 r f\left(\frac{2 x}{s}\right)-2 \gamma f(x)\right\|_{Y} \leq \varphi(x, x, 0) \text { para todo } x \in X
$$

sustituyendo $y=z=0$ y volvemos a reemplazar $x$ por $2 x$ en (2), obtenemos

$$
\left\|4 r f\left(\frac{2 x}{s}\right)-\gamma f(2 x)\right\|_{Y} \leq \varphi(2 x, 0,0)
$$

por (13) y (14), Obtenemos

$$
\begin{aligned}
\left\|\frac{f(2 x)}{4}-f(x)\right\|_{Y} & =\frac{1}{|4 \gamma|}\left\|2\left(2 r f\left(\frac{2 x}{s}\right)-2 \gamma f(x)\right)-\left(4 r f\left(\frac{2 x}{s}\right)-\gamma f(2 x)\right)\right\|_{Y} \\
\leq & \frac{1}{|4 \gamma|} \max \left\{|2| \cdot\left\|2 r f\left(\frac{2 x}{s}\right)-2 \gamma f(x)\right\|\left\|_{Y},\right\| 4 r f\left(\frac{2 x}{s}\right)-\gamma f(2 x) \|{ }_{Y}\right\} \\
\leq & \frac{1}{|4 \gamma|} \max \{\varphi(2 x, 0,0),|2| \varphi(x, x, 0)\}
\end{aligned}
$$

el resto de la pueba es similar a la prueba del Teorema 3.1.

Corolario 3.6 Sea $\theta$ un número real positivo y $q$ es un numero real con $q>2$. Sea $f: X \longrightarrow Y$ una aplicación con $f(0)=0$ y satisface (8). Entonces existe una única aplicación cuadrática $Q: X \longrightarrow Y$ tal que

$$
\|f(x)-Q(x)\|_{Y} \leq \frac{\left.|4| 2|2| \theta|| x\right|^{q}}{|4 \gamma|\left(|4|-|2|^{q}\right)} \text { para todo } x \in X
$$

Prueba . La prueba se utiliza el Teorema 3.5. tomando $\varphi(x, y, z)=\theta\left(\|x\|^{q}+\|y\|^{q}+\|z\|^{q}\right)$ para todo $x \in X$. Entonces podemos tomar $\alpha=|2|^{q-2}$ y obtenemos el resultado deseado .

Teorema 3.7 Sea $X$ un espacio normado No-Arquimediano e $Y$ un espacio completo NoArquimediano. Sea $\varphi: X^{3} \longrightarrow[0,+\infty)$ un funcional tal que existe un $\alpha<1$ con

$$
\varphi\left(\frac{x}{2}, \frac{y}{2}, \frac{z}{2}\right) \leq \frac{\alpha}{|4|} \varphi(x, y, z) \text { para todo } x, y, z \in X .
$$

Sea $f: X \longrightarrow Y$ una aplicación con $f(0)=0$ y satisface (2). Entonces existe una única aplicación cuadrática $Q: X \longrightarrow Y$ tal que

$$
\|f(x)-Q(x)\|_{Y} \leq \frac{\alpha \cdot \max \{\varphi(2 x, 0,0),|2| \varphi(x, x, 0)\}}{|4 \gamma|(1-\alpha)} \text { para todo } x \in X
$$

Prueba . Se deduce de la ecuación (15) que

$$
\left\|f(x)-4 f\left(\frac{x}{2}\right)\right\|_{Y} \leq \frac{1}{|\gamma|} \max \left\{\varphi(x, 0,0),|2| \varphi\left(\frac{x}{2}, \frac{x}{2}, 0\right)\right\} \leq \frac{\alpha}{|4 \gamma|} \max \{\varphi(2 x, 0,0),|2| \varphi(x, x, 0)\}
$$

La prueba es similar a la prueba del Teorema 3.1. y 3.5.

Corolario 3.8 Sea $\theta$ un número real positivo y $q$ es un número real con $0<q<2$. Sea $f: X \longrightarrow Y$ una aplicación con $f(0)=0$ y satisface (8). Entonces existe una única aplicación cuadrática $Q: X \longrightarrow Y$ tal que

$$
\|f(x)-Q(x)\|_{Y} \leq \frac{\left.|4| 2|2| \theta|| x\right|^{q}}{|4 \gamma|\left(|2|^{q}-|4|\right)} \text { para todo } x \in X
$$


Prueba . La prueba se utiliza el Teorema 3.7. tomando $\varphi(x, y, z)=\theta\left(\|x\|^{q}+\|y\|^{q}+\|z\|^{q}\right)$ para todo $x \in X$. Entonces podemos tomar $\alpha=|2|^{2-q}$ y obtenemos el resultado deseado .

Sea $f: X \longrightarrow Y$ una aplicación que satisface $f(0)=0$ y (1). Sea $f_{e}(x):=\frac{f(x)+f(-x)}{2}$ y $f_{o}(x):=\frac{f(x)-f(-x)}{2}$. Entonces la aplicación $f_{o}, f_{e}$ satisface (1) tal que $f(x)=f_{e}(x)+f_{o}(x)$. por otra parte

$$
\left\|D_{f_{o}}(x, y, z)\right\| \leq \frac{\max \left\{D_{f}(x, y, z), D_{f}(-x,-y,-z)\right\}}{|2|} \leq \frac{\max \{\varphi(x, y, z), \varphi(-x,-y,-z)\}}{|2|}
$$

$\mathrm{y}$

$$
\left\|D_{f_{e}}(x, y, z)\right\| \leq \frac{\max \left\{D_{f}(x, y, z), D_{f}(-x,-y,-z)\right\}}{|2|} \leq \frac{\max \{\varphi(x, y, z), \varphi(-x,-y,-z)\}}{|2|}
$$

Para todo $x, y, z \in X$ donde $D_{f}(x, y, z)$ es el operador de diferencia del funcional de la ecuación (1). Así obtenemos el siguiente Teorema

Teorema 3.9 Sea $X$ un espacio normado No-Arquimediano e $Y$ un espacio completo NoArquimediano. Sea $\varphi: X^{3} \longrightarrow[0,+\infty)$ un funcional tal que existe un $\alpha<1$ con

$$
\varphi(2 x, 2 y, 2 z) \leq|4| \alpha \varphi(x, y, z)
$$

para todo $x, y, z \in X$. Sea $f: X \longrightarrow Y$ una aplicación con $f(0)=0$ y satisface (3) . Entonces existe una única aplicación aditiva $A: X \longrightarrow Y$ y una única aplicación cuadrática $Q: X \longrightarrow Y$ tal que

$$
\begin{aligned}
\| f(x)-Q(x)- & A(x) \|_{Y} \leq \max \left\{\left\|\frac{f(x)-f(-x)}{2}-A(x)\right\|\left\|_{Y},\right\| \frac{f(x)-f(-x)}{2}-Q(x)\|\|_{Y}\right\} \\
\leq & \max \left\{\frac{\max \{\max \{\varphi(2 x, 0,0), \varphi(-2 x, 0,0)\}, \max \{\varphi(x, x, 0), \varphi(-x,-x, 0)\}\}}{|4 \gamma|(1-\alpha)},\right. \\
& \left.\frac{\max \{\max \{\varphi(2 x, 0,0), \varphi(-2 x, 0,0)\},|2| \max \{\varphi(x, x, 0), \varphi(-x,-x, 0)\}\}}{|8 \gamma|(1-\alpha)}\right\}
\end{aligned}
$$

para todo $x \in X$

Teorema 3.10 Sea $X$ un espacio normado No-Arquimediano e $Y$ un espacio completo NoArquimediano. Sea $\varphi: X^{3} \longrightarrow[0,+\infty)$ un funcional tal que existe un $\alpha<1$ con

$$
\varphi\left(\frac{x}{2}, \frac{y}{2}, \frac{z}{2}\right) \leq \frac{\alpha \varphi(x, y, z)}{|2|}
$$

para todo $x, y, z \in X$. Sea $f: X \longrightarrow Y$ una aplicación con $f(0)=0$ y satisface (3) . Entonces existe una única aplicación aditiva $A: X \longrightarrow Y$ y una única aplicación cuadrática $Q: X \longrightarrow Y$ tal que

$$
\begin{gathered}
\|f(x)-Q(x)-A(x)\|_{Y} \\
\leq \alpha \cdot \max \left\{\frac{\max \{\max \{\varphi(2 x, 0,0), \varphi(-2 x, 0,0)\}, \max \{\varphi(x, x, 0), \varphi(-x,-x, 0)\}\}}{|4 \gamma|(1-\alpha)},\right. \\
\left.\frac{\max \{\max \{\varphi(2 x, 0,0), \varphi(-2 x, 0,0)\},|2| \max \{\varphi(x, x, 0), \varphi(-x,-x, 0)\}\}}{|8 \gamma|(1-\alpha)}\right\}
\end{gathered}
$$

para todo $x \in X$ 


\section{Estabilidad del Funcional de la Ecuación (1): Método Directo}

Teorema 4.1 Sea $G$ un espacio vectorial y que $X$ es un espacio de Banach No-Arquimediano. Supongamos que $\varphi: G^{3} \longrightarrow[0,+\infty)$ una función tal que

$$
\lim _{n \longrightarrow \infty}|2|^{n} \varphi\left(\frac{x}{2^{n}}, \frac{y}{2^{n}}, \frac{z}{2^{n}}\right)=0 \text { para todo } x, y, z \in G
$$

Además, para algún $x \in G$, el limite

$$
\Omega(x)=\lim _{n \longrightarrow \infty} \operatorname{máx}\left\{|2|^{k} \operatorname{máx}\left\{\varphi\left(\frac{x}{2^{k}}, 0,0\right), \varphi\left(\frac{x}{2^{k+1}}, \frac{x}{2^{k+1}}, 0\right)\right\} ; 0 \leq k<n\right\}
$$

y existe $f: G \longrightarrow X$ es una aplicación que satisface

$$
\begin{array}{r}
\| r f\left(\frac{x+y+z}{s}\right)+r f\left(\frac{x-y+z}{s}\right)+r f\left(\frac{x+y-z}{s}\right)+r f\left(\frac{-x+y+z}{s}\right) \\
-\gamma f(x)-\gamma f(y)-\gamma f(z) \|_{X} \leq \varphi(x, y, z)
\end{array}
$$

Entonces el limite

$$
A(x):=\lim _{n \longrightarrow \infty} 2^{n} f\left(\frac{x}{2^{n}}\right)
$$

existe para cada $x \in G$ y definimos una aplicación aditiva $A: G \longrightarrow X$ tal que

$$
\|f(x)-A(x)\| \leq \frac{1}{|\gamma|} \Omega(x)
$$

En particular, si

$$
\lim _{j \longrightarrow \infty} \lim _{n \longrightarrow \infty} \operatorname{máx}\left\{|2|^{k} \operatorname{máx}\left\{\varphi\left(\frac{x}{2^{k}}, 0,0\right), \varphi\left(\frac{x}{2^{k+1}}, \frac{x}{2^{k+1}}, 0\right)\right\} ; j \leq k<n+j\right\}=0
$$

Entonces A es la única aplicación aditiva que satisface (22)

Demostración. Por (11), sabemos que

$$
\left\|f(x)-2 f\left(\frac{x}{2}\right)\right\|_{X} \leq \frac{1}{|\gamma|} \operatorname{máx}\left\{\varphi(x, 0,0) \varphi\left(\frac{x}{2}, \frac{x}{2}, 0\right)\right\}
$$

para todo $x \in G$. reemplazamos $x$ por $\frac{x}{2}$ en (23), obtenemos

$$
\left\|2^{n} f\left(\frac{x}{2^{n}}\right)-2^{n+1} f\left(\frac{x}{2^{n+1}}\right)\right\|_{X} \leq \frac{|2|^{n}}{|\gamma|} \operatorname{máx}\left\{\varphi\left(\frac{x}{2^{n}}, 0,0\right), \varphi\left(\frac{x}{2^{n+1}}, \frac{x}{2^{n+1}}, 0\right)\right\}
$$

por lo tanto se deduce (21) y (24) que la sucesión $\left\{2^{n} f\left(\frac{x}{2^{n}}\right)\right\}_{n \geq 1}$ es una sucesión de cauchy. desde que $X$ es completo, resulta que $\left\{2^{n} f\left(\frac{x}{2^{n}}\right)\right\}_{n \geq 1}$ es convergente.

$$
A(x)=\lim _{n \longrightarrow \infty} 2^{n} f\left(\frac{x}{2^{n}}\right)
$$

por inducción en $n$, primero se mostrará que

$$
\left\|2^{n} f\left(\frac{x}{2^{n}}\right)-f(x)\right\|_{X} \leq \frac{1}{|\gamma|} \operatorname{máx}\left\{|2|^{k} \operatorname{máx}\left\{\varphi\left(\frac{x}{2^{k}}, 0,0\right), \varphi\left(\frac{x}{2^{k+1}}, \frac{x}{2^{k+1}}, 0\right)\right\} ; 0 \leq k<n\right\}
$$

para todo $n \geq 1$ y $x \in G$. al tomar $n \longrightarrow \infty$ en (25) y (20). se obtiene (22) . por (19) y (21),obtenemos 


$$
\begin{aligned}
\| r A\left(\frac{x+y+z}{s}\right) & +r A\left(\frac{x-y+z}{s}\right)+r A\left(\frac{x+y-z}{s}\right)+r A\left(\frac{-x+y+z}{s}\right)-\gamma A(x)-\gamma A(y)-\gamma A(z) \|_{X} \\
= & \lim _{n \longrightarrow \infty}|2|^{n} \| r f\left(\frac{x+y+z}{2^{n} s}\right)+r f\left(\frac{x-y+z}{2^{n} s}\right)+r f\left(\frac{x+y-z}{2^{n} s}\right)+ \\
& \quad r f\left(\frac{-x+y+z}{2^{n} s}\right)-\gamma f\left(\frac{x}{2^{n}}\right)-\gamma f\left(\frac{y}{2^{n}}\right)-\gamma f\left(\frac{z}{2^{n}}\right) \|_{X} \\
\leq & \lim _{n \rightarrow \infty}|2|^{n} \varphi\left(\frac{x}{2^{n}}, \frac{y}{2^{n}}, \frac{z}{2^{n}}\right)=0
\end{aligned}
$$

para todo $x, y, z \in X$. por lo tanto, la aplicación $A: G \longrightarrow X$ satisface (1). Para demostrar la propiedad de unicidad de $A$, Sea $L$ otra aplicación que satisface (22). entonces tenemos

$$
\begin{aligned}
\|A(x)-L(x)\|_{X} & =\lim _{n \longrightarrow \infty}|2|^{n} \mid\left\|A\left(\frac{x}{2^{n}}\right)-L\left(\frac{x}{2^{n}}\right)\right\| \\
& \leq \lim _{n \rightarrow \infty}|2|^{n} \operatorname{máx}\left\{\left\|A\left(\frac{x}{2^{n}}\right)-f\left(\frac{x}{2^{n}}\right)\right\|\left\|_{X},\right\| f\left(\frac{x}{2^{n}}\right)-L\left(\frac{x}{2^{n}}\right) \|{ }_{X}\right\} \\
& \left.\leq \lim _{j \longrightarrow \infty} \lim _{n \rightarrow \infty} \operatorname{máx}\left\{|2|^{k} \cdot \operatorname{máx}\left\{\varphi\left(\frac{x}{2^{k}}\right), 0,0\right), \varphi\left(\frac{x}{2^{k+1}}, \frac{x}{2^{k+1}}, 0\right)\right\} ; j \leq k<n+j\right\} \\
& =0
\end{aligned}
$$

para todo $x \in G$. por lo tanto , $A=L$. esto completa la prueba.

Corolario 4.2 Sea $\xi:[0,+\infty) \longrightarrow[0,+\infty)$ es una función que satisface

$$
\xi\left(|2|^{-1} t\right) \leq \xi\left(|2|^{-1}\right) \xi(t), \xi\left(|2|^{-1}\right)<|2|^{-1}
$$

para todo $t \geq 0$. Asumimos que $\kappa>0$ y $f: G \longrightarrow X$ una aplicación con $f(0)=0$ tal que

$$
\begin{array}{r}
\| r f\left(\frac{x+y+z}{s}\right)+r f\left(\frac{x-y+z}{s}\right)+r f\left(\frac{x+y-z}{s}\right)+r f\left(\frac{-x+y+z}{s}\right)- \\
\gamma f(x)-\gamma f(y)-\gamma f(z) \|_{X} \leq \kappa(\xi\|x\|+\xi\|y\|+\xi\|z\|)
\end{array}
$$

para todo $x, y, z \in G$. Entonces existe una única aplicación aditiva $A: G \longrightarrow X$ tal que

$$
\|f(x)-A(x)\|_{X} \leq \frac{1}{|\gamma|} \operatorname{máx}\left\{\kappa \zeta|| x\left\|, \frac{2}{|2|} \kappa \zeta\right\| x \|\right\}
$$

Prueba . Definimos $\varphi: G^{3} \longrightarrow[0,+\infty)$ por $\varphi(x, y, z):=\kappa(\zeta\|x\|+\zeta\|y\|+\zeta\|z\|)$, entonces obtenemos

$$
\lim _{n \longrightarrow \infty} \varphi\left(\frac{x}{2^{n}}, \frac{y}{2^{n}}, \frac{z}{2^{n}}\right) \leq\left(|2| \xi|2|^{-1}\right)^{n} \varphi(x, y, z)=0
$$

para todo $x, y, z \in G$. La última igualdad del hecho de que $|2| \xi|2|^{-1}<1$. Por otra parte, se deduce que

$$
\begin{aligned}
\Omega(x) & =\lim _{n \longrightarrow \infty} \operatorname{máx}\left\{|2|^{k} \operatorname{máx}\left\{\varphi\left(\frac{x}{2^{k}}, 0,0\right), \varphi\left(\frac{x}{2^{k+1}}, \frac{x}{2^{k+1}}, 0\right)\right\} ; 0 \leq k<n\right\} \\
& \leq \operatorname{máx}\left\{\varphi(x, 0,0), \varphi\left(\frac{x}{2}, \frac{x}{2}, 0\right)\right\}=\operatorname{máx}\left\{\kappa \zeta\|x\|, \frac{2}{|2|} \kappa\|x\|\right\}
\end{aligned}
$$


existe para cada $x \in G$. Además, tenemos

$$
\begin{gathered}
\lim _{j \longrightarrow \infty} \lim _{n \longrightarrow \infty} \operatorname{máx}\left\{|2|^{k} \operatorname{máx}\left\{\varphi\left(\frac{x}{2^{k}}, 0,0\right), \varphi\left(\frac{x}{2^{k+1}}, \frac{x}{2^{k+1}}, 0\right)\right\} ; j \leq k<n+j\right\} \\
=\lim _{j \longrightarrow \infty}|2|^{j} \operatorname{máx}\left\{\varphi\left(\frac{x}{2^{j}}, 0,0\right), \varphi\left(\frac{x}{2^{j+1}}, \frac{x}{2^{j+1}}, 0\right)\right\}
\end{gathered}
$$

Luego , aplicando el Teorema 4.1.Queda demostrado

Teorema 4.3 Sea $G$ un espacio vectorial y $X$ un espacio de Banach No-Arquimediano. Supongamos $\varphi: G^{3} \longrightarrow[0,+\infty)$ es una función tal que

$$
\lim _{n \longrightarrow \infty} \frac{\varphi\left(2^{n} x, 2^{n} y, 2^{n} z\right)}{|2|^{n}}=0
$$

para todo $x, y, z \in G$. Además, para cualquier $x \in G$, el limite

$$
\Omega(x)=\lim _{n \longrightarrow \infty} \operatorname{máx}\left\{\frac{\operatorname{máx}\left\{\varphi\left(2^{k+1} x, 0,0\right), \varphi\left(2^{k} x, 2^{k} x, 0\right)\right\}}{|2|^{k}} ; 0 \leq k<n\right\}
$$

y existe $f: G \longrightarrow X$ una función que satisface (21). Entonces el limite $A(x)=\lim _{n \longrightarrow \infty} \frac{f\left(2^{n} x\right)}{2^{n}}$ existe para todo $x \in G$ y

$$
\|f(x)-A(x)\|_{X} \leq \frac{1}{|2 \gamma|} \Omega(x) \text { para todo } x \in G
$$

En particular, si

$$
\lim _{j \longrightarrow \infty} \lim _{n \longrightarrow \infty} \operatorname{máx}\left\{\frac{\operatorname{máx}\left\{\varphi\left(2^{k+1} x, 0,0\right), \varphi\left(2^{k} x, 2^{k} x, 0\right)\right\}}{|2|^{k}} ; j \leq k<n+j\right\}=0
$$

Entonces A es la única aplicación que satisface (29).

Prueba . Por (7), obtenemos

$$
\left\|\frac{f(2 x)}{2}-f(x)\right\|_{X} \leq \frac{\operatorname{máx}\{\varphi(2 x, 0,0), \varphi(x, x, 0)\}}{|2 \gamma|} \text { para todo } x \in G
$$

Reemplazando $x$ por $2^{n} x$ en (30) obtenemos

$$
\left\|\frac{f\left(2^{n+1} x\right)}{2^{n+1}}-\frac{f\left(2^{n} x\right)}{2^{n}}\right\|_{X} \leq \frac{\operatorname{máx}\left\{\varphi\left(2^{n+1} x, 0,0\right), \varphi\left(2^{n} x, 2^{n} x, 0\right)\right\}}{|2 \gamma| \cdot||^{n}}
$$

Así de (27) y (31) la sucesión $\left\{\frac{f\left(2^{n}\right)}{2^{n}}\right\}_{n \geq 1}$ es convergente.

$$
A(x):=\lim _{n \longrightarrow \infty} \frac{f\left(2^{n} x\right)}{2^{n}}
$$

por otro lado de (31) se tiene que

$$
\begin{aligned}
\left\|\frac{f\left(2^{p} x\right)}{2^{q}}-\frac{f\left(2^{q} x\right)}{2^{q}}\right\|_{X} & =\left\|\sum_{k=p}^{q-1} \frac{f\left(2^{k+1} x\right)}{2^{k}}-\frac{f\left(2^{k} x\right)}{2^{k}}\right\|_{X} \\
& \leq \operatorname{máx}\left\{\left\|\frac{f\left(2^{k+1} x\right)}{2^{k}}-\frac{f\left(2^{k} x\right)}{2^{k}}\right\|_{X} ; p \leq k<q-1\right\} \\
& \leq \frac{1}{|2 \gamma|} \operatorname{máx}\left\{\frac{\operatorname{máx}\left\{\varphi\left(2^{k+1} x, 0,0\right), \varphi\left(2^{k} x, 2^{k} x, 0\right)\right\}}{|2|^{k}} ; p \leq k<q\right\}
\end{aligned}
$$

para todo $x \in G$ y $p, q \geq 0$ con $q>p \geq 0$. si $p=0$, tomamos $q \longrightarrow \infty$ en la ecuación (28), nosotros obtenemos (29). el resto de la prueba es similar del Teorema 4.1. y esto completa la prueba. 
Teorema 4.4 Sea $G$ un espacio vectorial y $X$ un espacio de Banach No-Arquimediano. Supongamos que $\varphi: G^{3} \longrightarrow[0,+\infty)$ es una función tal que

$$
\lim _{n \longrightarrow \infty}|4|^{n} \varphi\left(\frac{x}{2^{n}}, \frac{y}{2^{n}}, \frac{z}{2^{n}}\right)=0
$$

para todo $x, y, z \in G$. Además, para cualquier $x \in G$, el limite

$$
\Theta(x)=\lim _{n \longrightarrow \infty} \operatorname{máx}\left\{|4|^{k} \operatorname{máx}\left\{\varphi\left(\frac{x}{2^{k}}, 0,0\right),|2| \varphi\left(\frac{x}{2^{k+1}}, \frac{x}{2^{k+1}}, 0\right)\right\} ; 0 \leq k<n\right\}
$$

y existe $f: G \longrightarrow X$ una función con $f(0)=0$ que satisface (21). Entonces el limite $Q(x)=\lim _{n \longrightarrow \infty} 4^{n} f\left(\frac{x}{2^{n}}\right)$ existe para todo $x \in G$ y define una aplicación cuadrática $Q: G \longrightarrow X$ tal que

$$
\|f(x)-Q(x)\|_{X} \leq \frac{1}{|\gamma|} \Theta(x)
$$

En particular, si

$$
\lim _{j \longrightarrow \infty} \lim _{n \longrightarrow \infty} \operatorname{máx}\left\{|4|^{k} \operatorname{máx}\left\{\varphi\left(\frac{x}{2^{k}}, 0,0\right),|2| \varphi\left(\frac{x}{2^{k+1}}, \frac{x}{2^{k+1}}, 0\right)\right\} ; j \leq k<n+j\right\}=0
$$

Entonces $Q$ es la única aplicación aditiva que satisface (34)

Prueba . Se tiene de (33) que

$$
\left\|f(x)-4 f\left(\frac{x}{2}\right)\right\|_{X} \leq \frac{1}{|\gamma|} \operatorname{máx}\left\{\varphi(x, 0,0),|2| \varphi\left(\frac{x}{2}, \frac{x}{2}, 0\right)\right\}
$$

reemplazando $x$ por $\frac{x}{2^{n}}$ en (35), obtenemos

$$
\left\|4^{n} f\left(\frac{x}{2^{n}}\right)-4^{n+1} f\left(\frac{x}{2^{n+1}}\right)\right\|_{X} \leq \frac{|4|^{n}}{|\gamma|} \operatorname{máx}\left\{\varphi\left(\frac{x}{2^{n}}, 0,0\right),|2| \varphi\left(\frac{x}{2^{n+1}}, \frac{x}{2^{n+1}}, 0\right)\right\}
$$

Se sigue de (32) y(36) que la suceción $\left\{4^{n} f\left(\frac{x}{2^{n}}\right)\right\}_{n \geq 1}$ es una sucesión de Cauchy. el resto de la prueba es similar de la demostración del Teorema 4.1.

Teorema 4.5 Sea $G$ un espacio vectorial y $X$ un espacio de Banach No-Arquimediano. Supongamos que $\varphi: G^{3} \longrightarrow[0,+\infty)$ es una función tal que

$$
\lim _{n \longrightarrow \infty} \frac{\varphi\left(2^{n} x, 2^{n} y, 2^{n} z\right)}{|4|^{n}}=0
$$

para todo $x, y, z \in G$.Además, para cualquier $x \in G$, el limite

$$
\Theta(x)=\lim _{n \longrightarrow \infty} \operatorname{máx}\left\{\frac{\operatorname{máx}\left\{\varphi\left(2^{k+1} x, 0,0\right), \varphi\left(2^{k} x, 2^{k} x, 0\right)\right\}}{|4|^{k}} ; 0 \leq k<n\right\}
$$

y existe $f: G \longrightarrow X$ una función con $f(0)=0$ que satisface (21). Entonces el limite $Q(x)=\lim _{n \longrightarrow \infty} \frac{f\left(2^{n} x\right)}{4^{k}}$ existe para todo $x \in G y$

$$
\|f(x)-Q(x)\|_{X} \leq \frac{1}{|4 \gamma|} \Theta(x) \text { para todo } x \in G
$$

En particular, si

$$
\left.\lim _{j \longrightarrow \infty} \lim _{n \longrightarrow \infty} \operatorname{máx}\left\{\frac{\operatorname{máx}\left\{\varphi\left(2^{k+1} x, 0,0\right), \varphi\left(2^{k} x, 2^{k} x, 0\right)\right.}{|4|^{k}}\right\} ; j \leq k<n+j\right\}=0
$$

Entonces $Q$ es la única aplicación aditiva que satisface (39). 
Sea $f: X \longrightarrow Y$ una aplicación que satisface $f(0)=0$ y $(1)$. Sea $f_{e}(x):=\frac{f(x)+f(-x)}{2}$ y $f_{o}(x):=$ $\frac{f(x)-f(-x)}{2}$. Entonces $f_{e}$ y $f_{o}$ son aplicaciones que satisfacen (1) talque $f(x)=f_{e}(x)+f_{o}(x)$. Por otra parte ,

$$
\left\|D_{f_{o}}(x, y, z)\right\| \leq \frac{\operatorname{máx} \varphi(x, y, z), \varphi(-x,-y,-z)}{|2|}
$$

$\mathrm{y}$

$$
\left\|D_{f_{e}}(x, y, z)\right\| \leq \frac{\operatorname{máx} \varphi(x, y, z), \varphi(-x,-y,-z)}{|2|}
$$

para todo $x, y, z \in X$, donde $D_{f}(x, y, z)$ es el operador diferencial de la función de la ecuación (1). Así obtenemos el siguiente Teorema.

Teorema 4.6 Sea $G$ un espacio vectorial y $X$ un espacio de Banach No-Arquimediano. Supongamos $\varphi: G^{3} \longrightarrow[0,+\infty)$ es una función tal que

$$
\lim _{n \longrightarrow \infty} \frac{\varphi\left(2^{n} x, 2^{n} y, 2^{n} z\right)}{|4|^{n}}=0
$$

para todo $x, y, z \in G$. Además

$$
\begin{array}{r}
\Omega^{*}(x)=\lim _{n \longrightarrow \infty} \operatorname{máx}_{0 \leq k<n}\left\{\operatorname { m a ́ x } \left\{\operatorname{máx}\left\{\varphi\left(2^{k+1} x, 0,0\right), \varphi\left(-2^{k+1} x, 0,0\right)\right\},\right.\right. \\
\left.\left.\operatorname{máx}\left\{\varphi\left(2^{k} x, 2^{k} x, 0\right), \varphi\left(-2^{k} x,-2^{k} x, 0\right)\right\}\right\} /|2|^{k+1}\right\}
\end{array}
$$

$y$

$$
\begin{array}{r}
\Theta^{*}(x)=\lim _{n \longrightarrow \infty} \operatorname{máx}_{0 \leq k<n}\left\{\operatorname { m a ́ x } \left\{\operatorname{máx}\left\{\varphi\left(2^{k+1} x, 0,0\right), \varphi\left(-2^{k+1} x, 0,0\right)\right\}\right.\right. \\
\left.\left., \operatorname{máx}\left\{\varphi\left(2^{k} x, 2^{k} x, 0\right), \varphi\left(-2^{k} x,-2^{k} x, 0\right)\right\}\right\} /\left(|2| \cdot|4|^{k}\right)\right\}
\end{array}
$$

existe para cada $x \in G$ y $f: G \longrightarrow X$ una función con $f(0)=0$ que satisface (21). Entonces existe una aplicación aditiva $A: G \longrightarrow X$ y una aplicación cuadrática $Q: G \longrightarrow X$ tal que

$$
\begin{gathered}
\|f(x)-A(x)-Q(x)\|_{X} \leq \operatorname{máx}\left\{\left\|\frac{f(x)+f(-x)}{2}-Q(x)\right\|_{X},\left\|\frac{f(x)-f(-x)}{2}-A(x)\right\|_{X}\right\} \\
\leq \operatorname{máx}\left\{\frac{1}{|2 \gamma|} \Omega^{*}(x), \frac{1}{|4 \gamma|} \Theta^{*}(x)\right\} \text { para todo } x \in G .
\end{gathered}
$$

En particular, si

$$
\begin{array}{r}
\lim _{j \longrightarrow \infty} \lim _{n \longrightarrow \infty} \operatorname{máx}_{j \leq k<n+j}\left\{\operatorname { m a ́ x } \left\{\operatorname{máx}\left\{\varphi\left(2^{k+1} x, 0,0\right), \varphi\left(-2^{k+1} x, 0,0\right)\right\},\right.\right. \\
\left.\left.\operatorname{máx}\left\{\varphi\left(2^{k} x, 2^{k} x, 0\right), \varphi\left(-2^{k} x,-2^{k} x, 0\right)\right\}\right\} /|2|^{k+1}\right\}=0
\end{array}
$$

$y$

$$
\begin{array}{r}
\lim _{j \longrightarrow \infty} \lim _{n \longrightarrow \infty} \operatorname{máx}_{j \leq k<n+j}\left\{\operatorname { m a ́ x } \left\{\operatorname{máx}\left\{\varphi\left(2^{k+1} x, 0,0\right), \varphi\left(-2^{k+1} x, 0,0\right)\right\},\right.\right. \\
\left.\left.\operatorname{máx}\left\{\varphi\left(2^{k} x, 2^{k} x, 0\right), \varphi\left(-2^{k} x,-2^{k} x, 0\right)\right\}\right\} /|2| \cdot|4|^{k}\right\}=0
\end{array}
$$

Entonces $A, Q$ son las únicas aplicaciones que satisface (40)

\section{Conclusión}

En este artículo vinculamos dos disciplinas diferentes como es el espacio de Banach No-Arquimedianos y Ecuaciones Funcionales. Hemos establecido la generalidad Hyers-Ulam estabilidad de la ecuación funcional (1) en espacios normados No-Arquimedianos. 


\section{REFERENCIAS BIBLIOGRÁFICAS}

[1] A.C.M. Van Rooij (1978). Non-Archimedean Fuctional Analysis. Marcel Dekker, Inc., New York.

[2] Alex Cruz H.(2007).El Teorema de Hahn Banach No-Arquimediano. Tesis de Licenciatura, UNFV. Lima .

[3] A.F. Monna (1970), Analyse Non-Archimedeanne. Springer-Verlag. New York, .

[4] Azadi Kenary H.(2011). Non-Archimedean stability of Cauchy-Jensen type functional equation. Int. J. Nonlinear Anal.

[5] Hensel K.(1897). Ubereine news begrundung der theorie der algebraischen Zahlen. Jahresber. Dtsch. Math.

[6] Hyers Dh. , ISAC G.(1998). RASSIAS TM. Stability of functional equations in several variables. Birkhauser, Basel.

[7] Peter Schneider .(2005). Nonarchimedean Fuctional Analysis New York, .

[8] Radu V.(2003). The fixed point alternative and the stability of functional equations. Fixed Point Theory.

[9] Schikhof W.(1984). Ultrametric calculus an introduction to p-adic análisis. Cambrigde University. 